\title{
Assessment of the Effect of Temporomandibular Joint Bony Ankylosis on Eustachian Tube Functions
}

\author{
Dehis Mohammad ${ }^{1}$, Nadia Kamal ${ }^{2}$, Samer Abbassi $^{1}$, Abeer Kamal ${ }^{3}$, Sayed Rashed ${ }^{4}$ \\ ${ }^{1}$ Department of Oral and Maxillofacial Surgery, Faculty of Oral \& Dental medicine, Cairo University, Cairo, Egypt \\ ${ }^{2}$ Department of Audiology, Faculty of Medicine, Ain Shams University, Cairo, Egypt \\ ${ }^{3}$ Department of Oral and Maxillofacial Surgery, College of Oral \& Dental Surgery, Misr University for Science \& Technology, Giza, Egypt \\ ${ }^{4}$ Department of Oral and Maxillofacial Surgery, Faculty of Oral \& Dental Medicine, Al Ahram Canadian University, Giza, Egypt
}

\section{Email address:}

mesd47@gmail.com (D. Mohamed),nadia.ainshams@gmail.com (N. Kamal),dds_s.abbassi@hotmail.com (S. Abbassi), abeermk7@gmail.com(A.Kamal),sayed_mody2001@yahoo.com (S. Rashed)

\section{To cite this article:}

Dehis Mohammad, Nadia Kamal, Samer Abbassi, Abeer Kamal, Sayed Rashed. Assessment of the Effect of Temporomandibular Joint Bony Ankylosis on Eustachian Tube Functions. International Journal of Clinical Oral and Maxillofacial Surgery. Vol. 3, No. 1, 2017, pp. 1-6. doi: $10.11648 /$ j.ijcoms.20170301.11

Received: March 19, 2017; Accepted: March 23, 2017; Published: April 18, 2017

\begin{abstract}
Objective: This study was designed to evaluate the effect of temporomandibular joint (TMJ) bony ankylosis on Eustachian tube (ET) functions. Subjects and Methods: Twelve patients suffering from TMJ bony ankylosis were selected from those attending the out-patient Clinic of Oral and Maxillofacial Surgery Department, Faculty of Oral and Dental Medicine, Cairo University. Twelve subjects (control group) underwent Eustachian tube function test were selected also. Tympanometry was utilized to assess Eustachian tube function. Three pressure reading (P1, P2, and P3) of the middle ear and external ear canal volume were recorded by the use of tympanometry. Comparisons were performed between patient and control group to evaluate the change of pressure. Results: Throughout the results, four ears showed type (C) tympanogram representing Eustachian tube dysfunction, two ears showed type (B) tympanogram which represent otitis media with effusion. While other ears showed type (A) tympanogram. On comparing the pressure values between the patients and the control, significant decrease was recorded in (P2) value in the patients group and no significant difference existed in the external canal volume. Conclusions: The Eustachian tube function is affected in patients of temporomandibular joint bony ankylosis, it cannot adapt to changes in pressure effectively. Patients of TMJ ankylosis are more liable to develop otitis media. Inflation-deflation test is a reliable method for evaluating Eustachian tube function in patients of (TMJ) ankylosis. The external ear canal volume was not affected in this group of patients.
\end{abstract}

Keywords: TMJ, Bony Ankylosis, Tympanometry, Eustachian Tube

\section{Introduction and Review of Literature}

Temporomandibular joint (TMJ) ankylosis usually impede the growth of upper and lower jaws. It ends up by osseous and soft tissue deformities. Impairments of orofacial function include the following, breathing problems due to the altered position of the larynx and the posterior position of the tongue, limited chewing ability, and alterations in speech, compromised oral hygiene and dental care, dental malocclusion, and psychological problem. [1,2] Review of literature concerning the TMJ ankylosis showed that this subject has received a considerable attention regarding etiology, epidemiology, diagnosis and treatment; however, revision of data has failed to detect the relation between TMJ ankylosis and Eustachian tube functions. The present study was planned in an attempt to evaluate the relation between the TMJ bony ankylosis and Eustachian tube (ET) functions.

The Eustachian tube acts as a pressure-equalizing valve for the middle ear; it is normally filled with air. When functioning properly, the Eustachian tube opens for a fraction of a second periodically in response to jaw movement, swallowing, chewing, yawning and Valsalva maneuver. It allows air into the middle ear to equalize pressure changes. Interference with this periodic opening and closing might 
result in hearing impairment and Eustachian tube dysfunction [3].

Tympanometry plays an important role in detecting middle ear pathology. The proposed study is intending to utilize the tympanometry as a method for evaluating Eustachian tube function. It has multiple advantages as compared with other methods. Tympanometry records the pressure inside the middle ear and induce changes in pressure in the external ear to assess the ability of Eustachian tube to function and adapt with these changes. In addition, tympanometry is planned to measure external ear canal volume to detect any possible deformities that may be induced by TMJ ankylosis. [4-6]

\section{Subjects and Methods}

The present study comprised twenty four Egyptian people, divided into two groups:

Group (A): Patient group, twelve adult patients, suffering from temporomandibular joint bony ankylosis. They were selected from those attending the out-patient clinic, Oral and Maxillofacial Surgery Department, Faculty of Oral and Dental Medicine, Cairo University.

Diagnosis of the TMJ bony ankylosis was established on the basis of careful history, clinical, radiographic examinations, and otological assessment to exclude obvious otological disorders.

Exclusion criteria: Fibrous ankylosis, extremes of age, patients with ear disease and patients with mental disorders.

Analysis of patient group:

Patient group included twelve individuals, five males $(41.6 \%)$ and seven females $(58.4 \%)$, with mean of 29.5 years. The mean of duration of the ankylosis was 19.25 years. Eight patients were suffering from bilateral ankylosis while it was unilateral in four patients, two of them on the left side and two on the right side. Trauma was the etiological factor in ten subjects $(83.3 \%)$ and two subjects were congenital (16.6\%). Chin scar was detected in ten cases. Bird face deformity was observed in eight patients additionally deviation of the mandible toward the affected side existed in three patients. Maximum interincisal opening (IIO) ranged from 0 to $22 \mathrm{~mm}$ with mean value of $5 \mathrm{~mm}$. seven cases showed recurrent (TMJ) ankylosis while five of them were not operated before.

Group (B): The control group, twelve adult healthy volunteer subjects, with no history of facial or head trauma or any ear disease they were selected with mean of age equal to patient group.

The entire sample was subjected to the following:

Otological examination: including inspection and recording for aural symptoms. Removing of ear wax was performed by ear droplet (Remowax: Carpamide peroxide $5 \%$, Hi pharm for manufacturing and pharmaceuticals and chemicals, Cairo, Egypt) and ear wash, if needed, in order to assure a clean external ear canal before evaluating Eustachian tube function.

Assessment of the Eustachian tube function by the tympanometer: (TITAN, Interacoustic, Denmark). The probe of the device is inserted into the external ear, it was snugly fit and air tight seal guaranteed. This was assured by green light of the device. Tone of $226 \mathrm{~Hz}$ was transmitted into the ear and the pressure on the external ear canal changes from $(-600$ to +300 ) deka Pascal ( $\mathrm{daPa}$ is a measurement of pressure). The results appear on the screen as a curve called tympanogram. The maximum tympanometric peak occurs when the pressure of the external auditory canal and the middle ear becomes equal. It is recorded on the device as (P1) that is mean middle ear pressure without stress. The height of the peak represents the impedance (mobility or stiffness) of the tympanic membrane. Tympanometer also provide a measurement of the external auditory canal volume (ml).

Baseline tympanogram (P1) was obtained as explained above, then inflation-deflation test was done by asking the patient to do valsalva maneuver by swelling the cheek by forced expiration with the mouth closed and the nostrils pinched, obtaining an air passage to the middle ear and the middle ear pressure was recorded as (P2) that is mean middle ear pressure with positive stress. After that the pressure in the external ear was increased to $(300 \mathrm{daPa})$ and asking the patient to swallow three times in order to equalize the middle ear pressure that increased. Then the middle ear pressure was recorded as (P3) that is mean middle ear pressure with negative stress.

The external ear canal volume (ECV) was measured automatically by the device and it represent the volume extending from the tip of the probe to the tympanic membrane in $\mathrm{ml}$.

During the test the patient must remain quiet and was asked not to talk during the procedure, the test was repeated if the patient has violated these instructions.

Patient group (A): were subjected to radiographic examination. It included panoramic view and computed tomography (CT) of the TMJ including axial, coronal cuts and three-dimensional reconstruction of the CT. The obtained images utilized to demonstrate ankylotic bony mass regarding its size and extent. Surgical intervention to treat TMJ ankylosis was performed. The patients were admitted in the Dental Educational Hospital, Cairo University to be operated under general anesthesia for releasing of ankylosis by gap arthroplasty.

Collection of data and statistical analysis: The data collected from the tympanometer (P1, P2, P3 and ECV) were subjected for tabulation, descriptive and analytical statistics using SPSS software version 20 for 2010- Chicago- USA. Mean and standard deviation were used. T-paired Student test for evaluation of the changes and $\mathrm{P}-$ value was significance if $\leq 0.05$.

The following comparisons were done:

I- Between the right and left ears of the control group regarding $(\mathrm{P} 1, \mathrm{P} 2, \mathrm{P} 3$ and $\mathrm{ECV})$ if no significance difference the sample will be considered containing 24 ears.

II- Between the right ears and left ears of the patient group regarding $(\mathrm{P} 1, \mathrm{P} 2, \mathrm{P} 3$ and $\mathrm{ECV})$ if no significance difference the sample will be considered 
containing 24 ears

III-Patient group (ankylosis) versus control group regarding (P1, P2, P3 and ECV)

IV-Patient group (ankylosis) versus control group regarding difference in middle ear pressure values ( $\mathrm{P} 1$ minus $\mathrm{P} 2$ and $\mathrm{P} 2$ minus $\mathrm{P} 3$ ) if the difference is less than $10 \mathrm{daPa}$; it indicates Eustachian tube dysfunction according to Hidir et al [7].

\section{Results}

\subsection{Comparison Between the Right and Left Ears of the Control Group Regarding (ECV and Middle Ear Pressure P1, P2, P3)}

Middle ear pressure of the right ear: The base line middle ear pressure (P1) of the right ear was ranged from (-41) to (50) with mean of (-6.16). All cases showed Type A tympanogram. The middle ear pressure with Valsalva maneuver (P2) was ranged from (-15) to (162) with mean of (29). The middle ear pressure after swallowing (P3) was ranged from (-48) to (69) with mean of (1.83).

Middle ear pressure of the left ear: The base line middle ear pressure (P1) of the right ear was ranged from $(-28)$ to (20) with mean of (-8.83). All cases showed Type A tympanogram. The middle ear pressure with Valsalva maneuver (P2) was ranged from (-10) to (172) with mean of (39.08). The middle ear pressure after swallowing (P3) was ranged from (-15) to (5) with mean of (-2.08).

External ear canal volume: Measurement of the external ear canal volume $(\mathrm{ECV})$ of the right ear, showed that the volume ranged from (0.8) to (1.3) with mean of (1.05). External ear canal volume (ECV) of the left ear, showed a range from (0.8) to (1.3) with mean of (1.04).

On comparing the middle ear pressure (P1, P2 and P3) and the external ear canal volume (ECV) between the right and the left ears of the control group, it was detected that there is no significant difference. The sample was considered to include 24 ears. Table 1.

Table 1. Statistical analysis between right and left ears of the control group as regard of $P 1, P 2, P 3$ and $E C V$.

\begin{tabular}{lllllll}
\hline & Side & $\mathbf{N}$ & Mean & Std. Deviation & T & P value \\
\hline P1 & Right & 12 & -6.1667 & 24.18427 & 0.325 & 0.749 \\
& Left & 12 & -8.8333 & 14.98990 & & \\
P2 & Right & 12 & 29.0000 & 51.46755 & -0.488 & 0.630 \\
& Left & 12 & 39.0833 & 49.67432 & & \\
P3 & Right & 12 & 1.8333 & 28.33832 & 0.468 & 0.645 \\
& Left & 12 & -2.0833 & 6.20056 & & \\
ECV & Right & 12 & 1.0508 & 0.16806 & 0.87 & 0.932 \\
& Left & 12 & 1.0450 & 0.16223 & & \\
\hline
\end{tabular}

3.2. Comparison Between the Right Ears and Left Ears of the Patient Group Regarding (ECV and Middle Ear Pressure P1, P2, P3)

Middle ear pressure of the right ear: The base line middle ear pressure (P1) of the right ear was ranged from $(-110)$ to (10) with mean of $(-20.25)$. Cases no. 5 and no. 6 showed type C tympanogram with readings (-65) and (-110) respectively. The other cases showed Type A tympanogram. (Table 2) P2 values pressure was range from $(-105)$ to $(25)$ with mean of $(-11.25)$. P3 values was ranged from $(-115)$ to (25) with mean of (-13.16).

Middle ear pressure of the left ear:

The base line middle ear pressure ( $\mathrm{P} 1)$ of the right ear was ranged from (-55) to (78) with mean of (1.8). Cases no. 6 and no. 9 showed type $\mathrm{C}$ tympanogram with reading of $(-55)$ and (78) respectively. Cases no. 4 and no. 12 showed Type B tympanogram with no peak (NP) and no pressure reading. (Table 2) The other cases showed Type A tympanogram. P2 values was range from (-55) to (15) with mean of (-5.7). P3 was ranged from (-55) to (50) with mean of (1.4).

Table 2. Distribution of the tympanograms according to Jerger's (8) classification.

\begin{tabular}{lll}
\hline Case No. & Right & Left \\
\hline 1 & A & A \\
2 & A & A \\
3 & A $^{*}$ & A \\
4 & A & B \\
5 & C & A \\
6 & C & C $^{*}$ \\
7 & A & A \\
8 & A & A \\
9 & A & C $^{*}$ \\
10 & A & A \\
11 & A* & A \\
12 & A & B \\
\hline
\end{tabular}

* Non ankylosis side.

$\%$ abnormal ear function.

Type B will excluded from the study as it indicate otitis media with effusion.

External ear canal volume: The external ear canal volume (ECV), of the right ear was ranged from (0.75) to (1.17) with mean of (1). External canal volume (ECV) of the left ear was range from (0.75) to (1.55) with mean of (1.13).

On comparing the middle ear pressure (P1, P2 and P3) and external ear canal volume (ECV) between the right ears and the left ears of the patients group, it was detected that there is no significant difference. The sample was considered to include 22 ears as type B tympanometer will exclude from the study because it indicate otitis media with effusion. Table 3.

Table 3. Statistical analysis between right and left ears of the patients group as regard of $P 1, P 2, P 3$ and $E C V$.

\begin{tabular}{lllllll}
\hline & Side & $\mathbf{N}$ & Mean & Std. Deviation & t & P value \\
\hline \multirow{2}{*}{ P1 } & Right & 12 & -20.2500 & 34.40963 & -1.536 & 0.140 \\
& Left & 10 & 1.8000 & 32.40988 & & \\
P2 & Right & 12 & -11.2500 & 36.82915 & -0.420 & .0679 \\
& Left & 10 & -5.7000 & 21.34401 & & \\
P3 & Right & 12 & -13.1667 & 35.87563 & -0.955 & 0.351 \\
& Left & 10 & 1.4000 & 35.29935 & & \\
EC & Right & 12 & 1.0000 & 0.11298 & -1.384 & 0.180 \\
V & Left & 12 & 1.1300 & 0.30523 & & \\
\hline
\end{tabular}




\subsection{Comparison Between Patient Group (Ankylosis) Versus Control Group Regarding (P1, P2, P3 and ECV)}

On comparing the middle ear pressure (P1, P2 and P3) and the external ear canal volume between patients and control group, it was detected that there is a significant difference in $\mathrm{P} 2$ Value with P-value of (0.001) and there is no significant difference in P1, P3 and ECV. Table 4. Figure 1.

Table 4. Comparison between patients and control as regard of $P 1, P 2, P 3$ and $E C V$.

\begin{tabular}{lllllll}
\hline & group & N & Mean & Std. Deviation & t & P value \\
\hline \multirow{2}{*}{ P1 } & patients & 22 & -10.2273 & 34.59278 & -0.332 & \multirow{2}{*}{0.741} \\
& control & 24 & -7.5000 & 19.72419 & & \\
P2 & patients & 22 & -8.7273 & 30.22799 & -3.485 & 0.001 \\
& Control & 24 & 34.0417 & 49.73449 & & \\
P3 & patients & 22 & -6.5455 & 35.54304 & -0.762 & 0.450 \\
& control & 24 & -0.1250 & 20.16090 & & \\
\multirow{2}{*}{ ECV } & patients & 22 & 1.0650 & 0.23467 & 0.294 & 0.770 \\
& Control & 24 & 1.0479 & 0.16157 & & \\
\hline
\end{tabular}

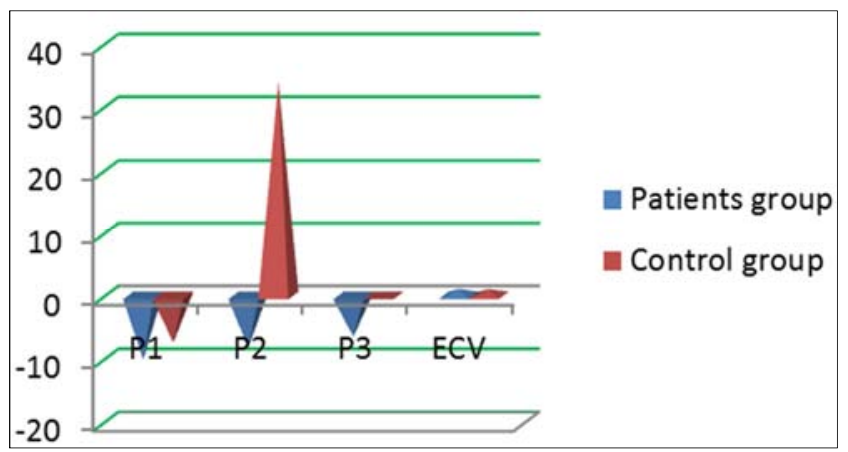

Figure 1. Mean of (P1, P2, P3 and ECV) in the patients and control group.

\subsection{Patient Group (Ankylosis) Versus Control Group Regarding Difference in Middle Ear Pressure) Values (P1 Minus P2 and P2 Minus P3)}

On comparing the difference in the middle ear pressure (P1-P2 and P2-P3) between patients and control group, it was detected that there is a significant difference in the changes in (P1-P2) and changes in (P2-P3) with P-value of (0.002) and (0.006) respectively. Table 5. Figure 2.

Table 5. Comparison between the patients and the control as regard of the changes in $P 1, P 2$ and $P 3$ values.

\begin{tabular}{lllllll}
\hline & group & $\mathbf{N}$ & Mean & Std. Deviation & t & P value \\
\hline P1- & patients & 22 & -1.5000 & 21.72940 & \multirow{2}{*}{3.263} & \multirow{2}{*}{0.002} \\
P2 & control & 24 & -41.5417 & 53.63239 & & \\
P2- & patients & 22 & -2.1818 & 19.99675 & -2.865 & 0.006 \\
P3 & Control & 24 & 34.1667 & 56.30790 & & \\
\hline
\end{tabular}

\section{Discussion}

The present study was planned in an attempt to evaluate the relation between the temporomandibular joint (TMJ) bony ankylosis and Eustachian tube (ET) functions. Loss of jaw movement by the ankylosis may alter the function of (ET) and this has created the hypothesis for necessity of this research for clarification. The obtained data will augment the field of oral and maxillofacial surgery by adding the dimension of audiology for cases of bony ankylosis.

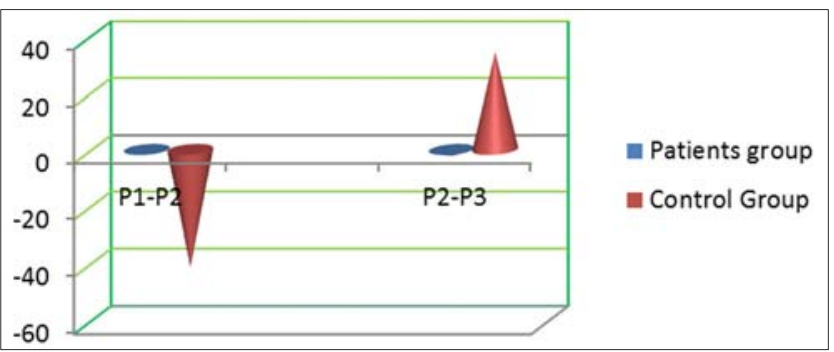

Figure 2. Mean of changes in middle ear pressure in the patients and control group.

Results of P1 (base line middle ear pressure) tympanometer were interpreted using Jerger [8] classification being used by several researchers [8-11] and it allows the present results to be calibrated and evaluated with other results in the field of audiology. In the current study P1 results showed type (C) category in 4 ears (16.6\%) which means marked Eustachian tube dysfunction. Tympanogram type (B) was identified for 2 ears $(8.4 \%)$ with no compliance peak which represent middle ear effusion. Tympanogram type (A) was found in (75\%). Additional investigation showed that although most cases were type (A), however Eustachian tube did not respond effectively to changes in pressure as normal healthy person. [9-10]

Inflation-deflation test was carried out to record P2 (middle ear pressure with Valsalva maneuver) and P3 (middle ear pressure after swallowing). This test illustrates the ability of Eustachian tube to function and provide ventilation to the middle ear with induced pressure and it was recommended to test Eustachian tube function by many authors [7-11]. Valsalva maneuver creates a positive pressure and a good functioning Eustachian tube should open and adapt with this changed pressure. [12] The current study showed significant difference in (P2) value and the patients could not fulfill effective tubal opening.

The present study revealed that Eustachian tube in patients suffering from temporomandibular joint bony ankylosis does not open effectively to adapt with the induced changes in pressure. This was noticed as the mean of changes from $\mathrm{P} 1$ to $\mathrm{P} 2(\mathrm{P} 1-\mathrm{P} 2)$ was $(-1.50 \mathrm{daPa})$ in the patients group and it was $(-41.54 \mathrm{daPa})$ in the control group with statistically significant difference. The same statistically significant difference was observed in the mean of changes from P2 to P3 (P2-P3) was (-2.81 daPa) in the patients group and it was $(34.16 \mathrm{daPa})$ in the control group. These findings are in accordance with other researchers reported Eustachian tube dysfunction in non-ankylosing (TMJ) disorders. [13- 16]

Detected aural symptoms in the patients suffering from temporomandibular joint ankylosis (ear pain, tinnitus, degree of hearing loss and ear fullness) may be attributed to embryological factor; the (TMJ) and the ear begin to develop in the same phase and that the ossicular chain of the middle ear muscles belong embryologically to the masticatory apparatus and later they become and serve as the hearing 
sense, hence disorder of the (TMJ) might have a reasoning effect on explanation Eustachian tube dysfunction. This concept is supported by researches of Rodriguez [17] and whyte. [18]

Close anatomical inter-relation between the middle ear and the TMJ create a reciprocal affection. Middle ear infection as otitis media was reported long time ago as an etiological factor for TMJ ankylosis [19, 20]. Bony ankylosis usually disturbs the anatomy due to exorbitant bone formation, it might extend to involve structures of the temporal bone; this abnormal bone formation may encroach on the tympanic plate, hence disturbed volumetric anatomy and decrease middle ear volume are induced. The resultant changes will affect the aeration of the middle ear and incite Eustachian tube dysfunction. This opinion is in agreement with the reports by Tuz [21] et al and Dehis [22] et al.

Hyper tonicity and isometric contraction of the muscles of mastication due to long standing ankylosis may lead to spastic tensor veli palatini; so it's normal function of opening the Eustachian tube will be impaired, the sequel is Eustachian tube dysfunction; this explanation is concordant with findings of Greene [23] about spastic tensor veli palatini.

The motor root of the trigeminal nerve supplies eight muscles. Two of them are tensor veli palatini and tensor tympani which are related to middle ear audiology and audiological problems. Long standing masticatory dysfunction due to ankylosis may affect motor impulses conducted via the motor root of the trigeminal nerve. This explanation needs further investigation particularly in patients with long standing ankylosis.

It has been reported that the pharyngeal and the laryngeal muscles have a role in activation the tensor tympani muscle and consequently the tympanic membrane. The reciprocal contraction of tensor tympani creates an air pump which helps in opening of Eustachian tube [24, 25]. It seems that TMJ bony ankylosis affects the function of the pharyngeal and laryngeal muscles and consequently Eustachian tube ventilation is affected. These finding is supported by the reports of Alaa [26] who reported affection of the upper airway volume in bony ankylosis cases.

Eustachian tube dysfunction in (TMJ) ankylosis might be due to the neighboring compression of the upper head of the medial pterygoid muscle. The result of this compression and punching on muscular mass of the tensor veli palatini (TVP) leads to failure in the role of (TVP) in opening the Eustachian tube inducing its dysfunction. This opinion is supported by McDonnell [27] and Leuwer [28] who identified the role of the medial pterygoid muscle in developing (ET) dysfunction and Kumar [29] et al who detected increased thickness of the medial pterygoid muscle in TMJ bony ankylosis patients.

External ear canal volume (ECV) has not been affected by temporomandibular joint bony ankylosis. This was revealed by the results of the current study, there was non-significant difference in (ECV) between the patient and control group. This finding can be justified as; despite the anatomical adjacency, the ankylotic bony mass tends to extend more medially to affect the middle ear rather than posteriorly and laterally. This is in concurrence with research of Dehis et al [22] that detected decreasing in the volume of the middle ear in patients suffering from TMJ ankylosis. Yet more accurate measures for (ECV) such as CT are needed in the future researches.

The effect of temporomandibular joint bony ankylosis on Eustachian tube seems to be multifactorial and needs further investigations. The present study recommends preoperative audiological assessment including Eustachian tube function to document any alteration due to TMJ ankylosis.

\section{References}

[1] El-Sheikh MM, Medra AM \& Warda MH: Bird face deformity secondary to bilateral temporomandibular joint ankylosis. J Craniomaxillofac Surg. 1996: 24; 96-103.

[2] Mcfadden R. \& Rishiraj B.: Treatment of temporomandibular joint ankylosis. J Can Dent Assoc. 2001: 67; 659-63.

[3] Seibert W. \& Danner J.: Eustachian tube function and the middle ear. Otolaryngol Clin North Am: 2006: 39; 1221-35.

[4] Williams B, Taylor BA, Clifton N. \& Bance M: Balloon dilation of the Eustachian tube: a tympanometric outcomes analysis. J Otolaryngol Head Neck Surg. 2016: 45; 13.

[5] Kumazawa T, Iwano T, Ushiro K, Kinoshita T, Hamada E. \& Kaneko A: Eustachian tube function tests and their diagnostic potential in normal and diseased ears. Acta Otolaryngol Suppl. 1993: 500; 10-3.

[6] Liang M., Zheng Y., Zhang Z., Xu Y., Qu Y., Chen S., Yang H., Haung Q., Qiu Z. \& Chen L.: Eustachian tube balloon dilation in Eustachian tube dysfunction related diseases. Chinese PMID. 2014: 28: 1759-61.

[7] Hidir Y., Ulus S., Karahatay S. \& Satar B.: A. comparative study on efficiency of middle ear pressure equalization techniques in healthy volunteers. Auris Nasus Larynx. 2011: $38 ; 450-5$.

[8] Jerger J.: Clinical experience with impedance audiometry. Arch Otolaryngol, 1970: 92; 311-24.

[9] Bredfeldt RC: An Introduction to Tympanometry. Am Fam Physician. 1991: 44; 2113-8.

[10] Moody SA, Alper CM \& Doyle WJ: Daily tympanometry in children during the cold season: association of otitis media with upper respiratory tract infections. Int $\mathrm{J}$ Pediatr Otorhinolaryngol. 1998: 45; 143-50.

[11] Charles D.: Eustachian tube: structure, function, role in otitis media. Charles D. Bluestone, Maria B. Bluestone PMPH-USA page 62, 2005.

[12] Swarts JD, Alper CM, \& Mandel EM. Villardo R. \& Doyle WJ: Eustachian tube function in adults without middle ear disease. Ann Otol Rhinol Laryngol. 2011: 120; 220-5.

[13] Sobhy OA, Koutb AR, Abdel-Baki FA, Ali TM., El Raffa IZ \& Khater AH: Evaluation of aural manifestations in temporomandibular joint dysfunction. Clin Otolaryngol Allied Sci. 2004: $29 ; 382-5$. 
[14] Penkner K, Keinz J, Schied W, Schied G. \& Lorenzoni M: The function of tensor veli palatine muscles in patients with aural symptoms and temporomandibular disorder. J Oral Rehabil. 200: 27; 344-8.

[15] De Felacio CM, Melchior Mde O, Ferreira CL \& Da Silva MA: Otologic symptoms of temporomandibular disorder and effect of orofacial myofunctional therapy. Cranio. 2008: 26; $118-25$.

[16] Keersmaekers K, De Boever JA. \& Van Den Berghe L: Otalgia in patients with temporomandibular joint disorders. J Prosthet Dent. 1996: 75; 72-6.

[17] Rodriguez Vazquez JF, Merida Velasco JR \& Jimenez Collado $\mathrm{J}$ : Relationships between the temporomandibular joint and the middle ear in human fetuses. J Dent Res. 1993: 72; 62-6.

[18] Whyte JR, González L, Cisneros AI, Yus C, Torres A \& Sarta R: Fetal Development of the Human Tympanic Ossicular Chain Articulations. Cells Tissues Organs. 2002: 171; 241-9.

[19] Kazanjian VH: Temporomandibular ankylosis. Am J Surg, 1955: 90: 905-10.

[20] El-Mofty S: Ankylosis of temporomandibular joint. Oral Surg. 1972: 33; 650-60.

[21] Tuz HH, Onder EM \& Kisnisci RS: Prevalence of otologic complaints in patients with temporomandibular disorder. Am J Orthod Dentofacial Orthop. 2003: 123; 620-3.

[22] Dehis M, Tantawy W, Kamal A, Rashed S \& Sayed M: Effect of temporomandibular joint bony ankylosis and surgical sequelae of gap arthroplasty on middle ear volume. Egyptian journal of oral \& maxillofacial surgery. 2013: 4; 72-8.

[23] Greene S. \& Laskin M.: Temporomandibular disorders: Moving from a dentally based to a medically based model. J Dental Res. 2000: 79; 1736-9.

[24] Zipfel TE, Kaza SR \& Greene JS: Middle-ear myoclonus. J Laryngol Otol. 200: 114; 207-9.

[25] Ishijima K, Sando I, Balaban CD, Miura M \& Takasaki K: Functional anatomy of levator veli palatini muscle and tensor veli palatini muscle in association with Eustachian tube cartilage. Ann Otol Rhinol Laryngol. 2002: 111; 530-6.

[26] Alaa A: Effect of gap arthroplasty on the upper airway in patients of temporomandibular joint ankylosis. Master thesis, Cairo University. 2000.

[27] McDonnell JP, \& Needleman HL, Charchut S, Allred, EN, Roberson DW, Kenna MA \& Jones D: The Relationship between dental overbite and Eustachian Tube dysfunction. Laryngoscope. 2001: 111; 310-6.

[28] Leuwer R, Schubert R, Kucinski T, Liebig T \& Maier H: The muscular compliance of the auditory tube: a model-based survey. Laryngoscope. 2002: 112; 1791-5.

[29] Kumar VV, Malik NA, Visscher CM, Ebenezer S, Sagheb K \& Lobbezoo F: Comparative evaluation of thickness of jawclosing muscles in patients with long-standing bilateral temporomandibular joint ankylosis. Clin Oral Investig. 2015: $19 ; 421-7$. 